\title{
Desarrollo de una cadena de suministro para la Central de Productores Agropecuarios de Amazonas (CEPROAA) - 2017
}

\section{Development of a supply chain for the Central Agricultural Producers of Amazonas (CEPROAA) - 2017}

\author{
Yuri Reina Marín
}

\section{RESUMEN}

El precio internacional del cacao se determina en los mercados de futuros de Londres y Nueva York. Su comercialización está organizada alrededor de contratos forward y opciones. El manejo agronómico de una plantación incluye el conjunto de prácticas que deben efectuarse durante toda su vida útil. Para los países productores es una importante actividad económica, social y cultural que se desarrolla en una dinámica familiar. Su cultivo depende de las condiciones agroecológicas de las zonas productoras. El problema planteado es si el desarrollo de una cadena suministro para la Central de Productores Agropecuarios de Amazonas permitirá mejorar los ingresos y reducir los costos involucrados. La muestra fue de 79 socios de un total de 439 socios activos. Los métodos utilizados fueron la revisión de documentos, archivos, entrevistas y encuestas. Como resultados se sabe que la mayor parte de los socios tienen más de 5 años de permanencia y su experiencia es mayor a 8 años, el rendimiento que tienen el $82 \%$ de socios oscila entre 642 y 1003 kilos, la mayoría de ellos usa el canal de regadío, se comercializa principalmente sin procesar, dentro de los factores que se deben mejorar figura los canales de comercialización y la gestión.

Palabras clave: Cacao, cadena de suministro, gestión, estrategia, comercialización.

\begin{abstract}
The international price of cocoa is determined in the futures markets of London and New York. Its marketing is organized around forward contracts and options. The agronomic management of a plantation includes the set of practices that must be carried out throughout its useful life. For producing countries, it is an important economic, social and cultural activity that develops in a family dynamic. Its cultivation depends on the agro-ecological conditions of the producing areas. The problem posed is whether the development of a supply chain for the Central Agricultural Producers of Amazonas will improve income and reduce the costs involved. The sample was 79 partners out of a total of 439 active members. The methods used were the review of documents, files, interviews and surveys. As results know that most of the partners have more than 5 years of permanence and their experience is greater than 8 years, the performance that $82 \%$ of partners have ranges between 642 and 1003 kilos, most of them use the channel irrigated, it is marketed mainly unprocessed, within the factors that must be improved figure marketing channels and management.
\end{abstract}

Keywords: Cocoa, supply chain, management, strategy, marketing.

\footnotetext{
Ingeniero Industrial, Profesor auxiliar a Tiempo completo de la Facultad de Ingeniería Zootecnista, Biotecnología y Agronegocios de la Universidad Nacional Toribio Rodríguez de Mendoza de Amazonas; especialista en cadena de suministro, Correo electrónico: yuri.reina@untrm.edu.pe
} 


\section{INTRODUCCIÓN}

La producción de cacao está altamente concentrada en unos pocos países no desarrollados. Según la $\operatorname{ICCO}$ (2012, p. 32), los principales productores en el ámbito mundial en el período 2012 son: Costa de Marfil con $1410 \mathrm{~m} \mathrm{t}$ m, Ghana con $890 \mathrm{~m} \mathrm{t} \mathrm{m}$ e Indonesia con $500 \mathrm{~m} \mathrm{t} \mathrm{m}$, los tres países produjeron el $86.2 \%$ del total mundial. En América Latina, los principales productores son Brasil con $190 \mathrm{~m} \mathrm{t} \mathrm{m} \mathrm{y}$ Ecuador con $175 \mathrm{~m} \mathrm{t}$ m; Perú logró $57.9 \mathrm{~m} \mathrm{t} \mathrm{m}$. Es sencillo, identificar que los principales productores son países en desarrollo, que se caracterizan por un desarrollo básico de su agricultura. La transformación industrial se da en los países desarrollados. El crecimiento de consumo del chocolate tiene cada vez mayor demanda, principalmente del cacao de fino de aroma y sabor, en alrededor del $10 \%$ al año debido al ingreso de consumo en el hábito alimenticio de países como Japón, China, entre otros, bajo el criterio de que el chocolate tiene propiedades anticancerígenas, antioxidante y anti estresante. Por esa razón el cultivo de cacao fino aromático tiene futuro en el mercado mundial, pero la tendencia es diversificar los sellos decertificación y los mercadeos (orgánico, solidario, fino aromático y los de origen o marca). La región Amazonas cuenta con todas las condiciones edafoclimáticas para ser una de las principales regiones productoras de cacao en el país. Amazonas alberga una gran biodiversidad de variedades nativas que se caracteriza por tener una gran diversidad de clones promisorios (Zamora, 2011). En el comercio del cacao existen dos mercados. El primero de ellos es el tradicional o de granos convencionales; el segundo corresponde al de granos especializados, en donde se manejan precios diferenciados. El primero es el mercado de los grandes volúmenes, el segundo muestra una alta tasa de crecimiento y su demanda se debe a que los granos para los consumidores tienen características especiales distintas a los convencionales (Barrientos, 2015). La cadena de suministro del cacao, sigue un recorrido similar al del café en términos geográficos, debido a que ambos necesitan de condiciones climatológicas muy parecidas. En ese sentido, los nodos de producción del cacao son San Martín, Ayacucho, Amazonas, Cusco y Huánuco y el principal punto de exportación es el puerto de Callao (Banco Mundial ,2016).

\section{MATERIAL Y MÉTODO}

\section{Diseño de la investigación}

El desarrollo de la cadena de suministro del cacao implica el conocimiento de todo el sistema relacionado a esta actividad, el cual implica los siguientes aspectos:

Diseño de la red de la cadena de suministro.

Planeación de la demanda y la oferta de la cadena de suministro.

Planeación y administración de inventarios en la cadena de suministro.

Diseño y planeación de la red de transporte.

Administración de la cadena de suministro.

\section{Población, muestra y muestreo}

\section{Población:}

Para el presente proyecto de investigación se trabó con la Central de Productores Agropecuarios de Amazonas - CEPROAA, que cuenta con 439 socios activos.

\section{Muestra:}

Para determinar la muestra de la investigación se tomó como base a cuatrocientos treinta y nueve socios activos y a través de la fórmula estadística de muestreo se determinó el tamaño de la misma.

$$
\mathrm{n}=\frac{N * Z_{\propto}^{2} * p * q}{d^{2} *(N-1)+Z_{\propto}^{2} * p * q}
$$

\section{Donde:}

n: Tamaño de la muestra

$N$ : Total de la población

$\boldsymbol{Z}_{\propto}$ : Nivel de confianza

p: Variabilidad positiva

$q$ : Variabilidad negativa (1-p)

$d$ : Grado de error

$$
\begin{gathered}
\mathrm{n}=\frac{439 * 1.96^{2} * 0.05 * 0.95}{0.05^{2} *(439-1)+1.96^{2} * 0.05 * 0.95} \\
=79
\end{gathered}
$$

De acuerdo a estos resultados se entrevistarán a 79 personas entre directivos, socios, comercializadores y productores que están involucrados con esta actividad agrícola.

\section{Fuentes de Información.}

La información para este estudio fue obtenida de las Agencias desconcentradas de agricultura, la 
Dirección de Competitividad Agraria, La Biblioteca "Padre Blas Valera"-UNTRM, la Central de Productores Agropecuarios de Amazonas (CEPROAA).

\section{Método de recolección de datos}

Para la obtención de datos se tuvo que viajar al Distrito de Cajaruro, Provincia de Uctubamba, con el fin de entrevistarse con los dirigentes de la Central de Productores Agropecuarios de Amazonas (CEPROAA). Esto permitió un acercamiento directo con el objeto de estudio, La entrevista con los asociados fue estructurada y guiada por preguntas específicas de una ficha técnica, cuyo objetivo fue levantar información concisa de la actividad productiva. Las preguntas cerradas ahorraban el tiempo de la entrevista y la divagación en respuestas incongruentes que se hubiesen podido dar. También se hizo la indagación en relación a la cadena productiva y comercial del cacao.

\section{Técnicas}

Las técnicas utilizadas fueron:

Entrevistas

Observación sistemática y no sistemática

Análisis de documentos

Relatos

\section{Instrumentos}

Se utilizó la base de datos de la Dirección Regional Agraria Amazonas, referente a la producción de Cacao.

\section{Procedimientos}

En cuanto al procedimiento de recolección de datos se tuvo en cuenta las siguientes etapas:

Se seleccionó y elaboró el instrumento de medición teniendo en cuenta la operacionalización de las variables.

El instrumento se aplicó a los actores involucrados en la presente investigación, esta se realizó en forma colectiva por exigencias, fines y propiedad de la investigación.

Como última etapa del proceso de recolección de datos se elaboraron la tabulación de las mediciones obtenidas para su respectivo análisis estadístico.

\section{RESULTADOS}

Tiempo como socio

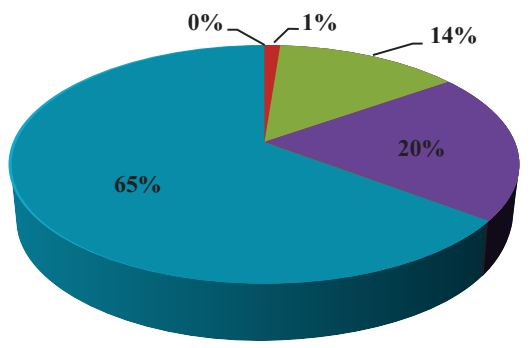

" 3-6 MESES 6-1 AÑO 1-3 AÑOS 3-5 AÑOS MÁS DE 5 AÑos

Figura 1.Tiempo como socio de la Asociación Central de Productores Agropecuarios de Amazonas-CEPROAA

Tiempo de actividad agrícola

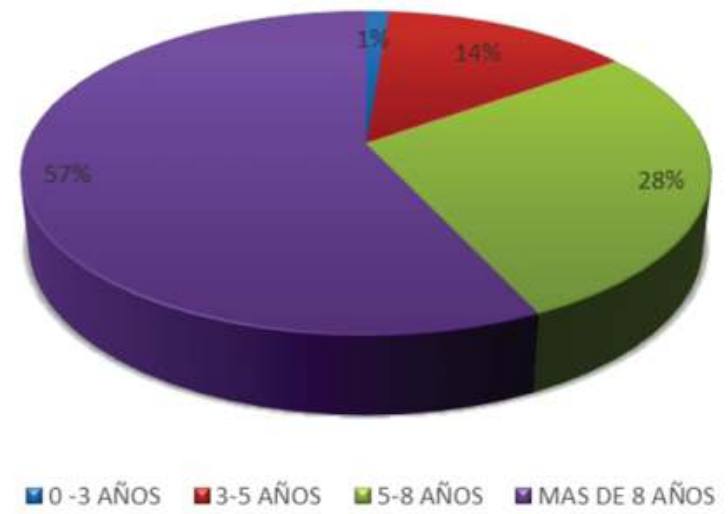

Figura 2. Tiempo de actividad agrícola.

Rendimiento de cacao por hectárea en $\mathrm{Kg}$.

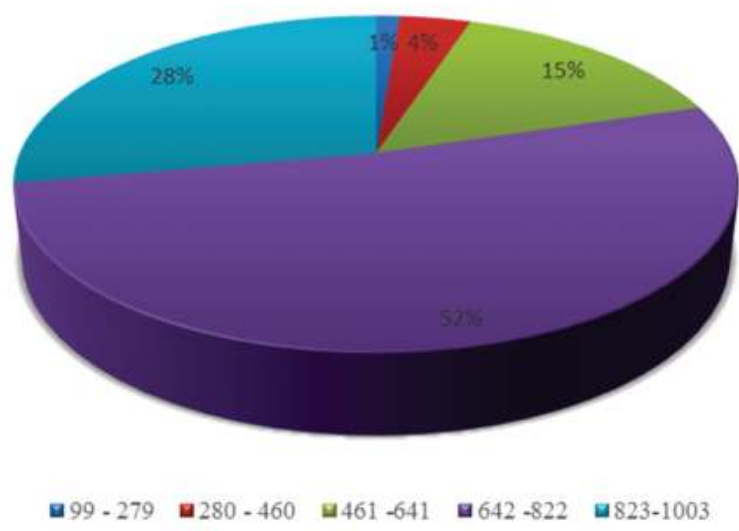

Figura 3.Rendimiento de cacao por hectárea. 


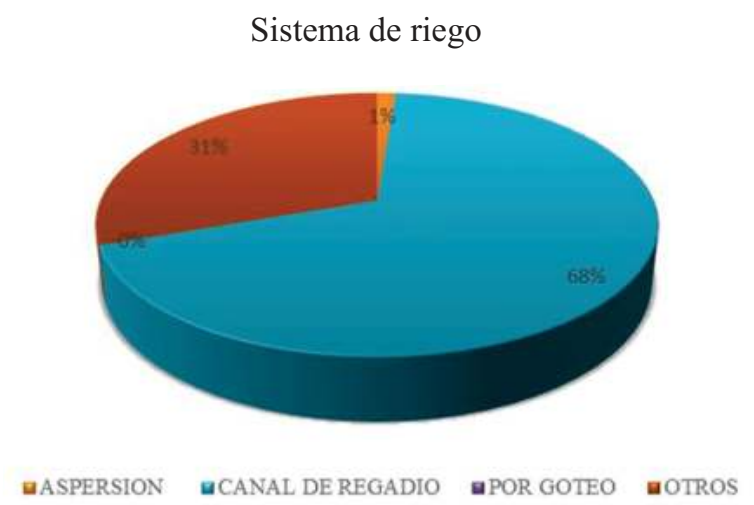

Figura 4. Tipos de sistema de riego.

Frecuencia de fertilización por año

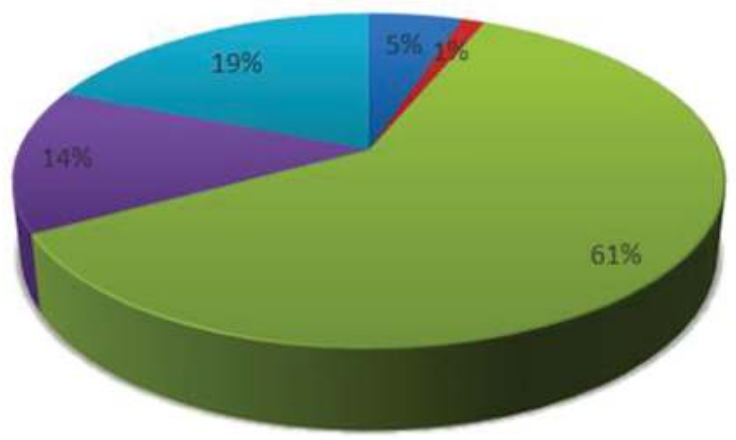

घ Kg. $0.5 \mathrm{Kg} . \square 1 \mathrm{Kg} . \square 2 \mathrm{Kg}$. $1 \mathrm{a} 2 \mathrm{Kg}$.

Figura 5. Frecuencia de uso de fertilizantes al año.

Forma de comercialización del cacao

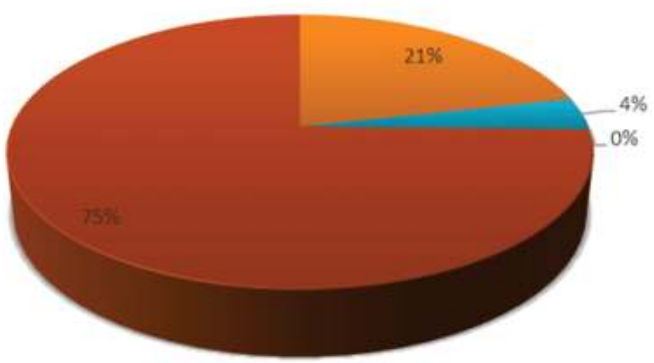

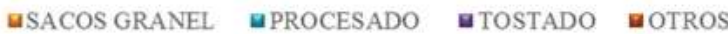

Participación en feria

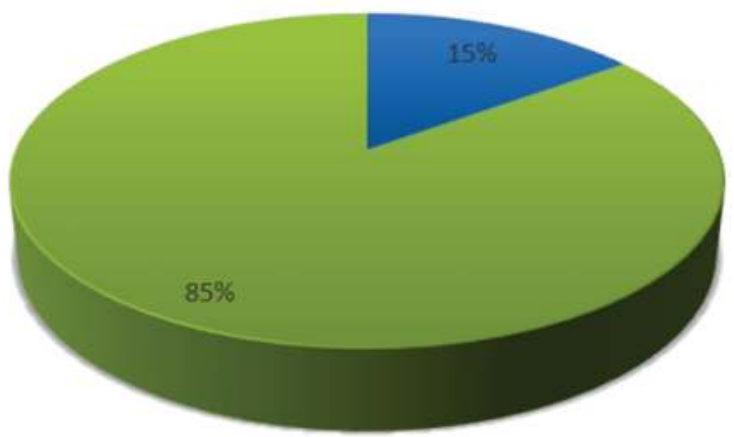

$\square \mathrm{SI} \boxminus \mathrm{NO}$

Figura 7.Participación en ferias.

Quien determina el precio del cacao

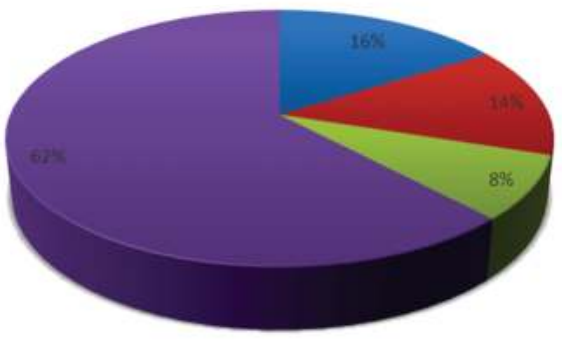

-MAYORISTA অACOPIADOR MPRODUCTOR MMERCADOEXTERIOR

Figura 8. Determinación del precio del cacao.

Factores a mejorar en la comercialización del cacao

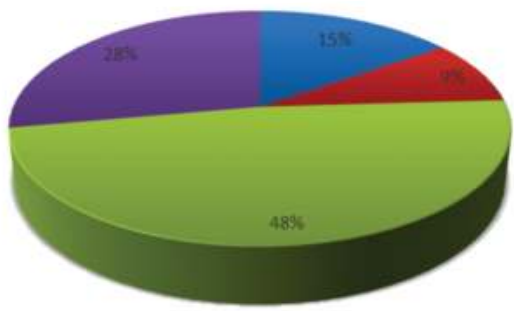

MEIOR GESTIONASOCIACION

a CREACION DE UNA MARCA ICANALES DE COMERCIALIZACION aOTROS

Figura 9.Factores a mejorar en la comercialización del cacao.

Figura 6. Forma de comercialización de cacao. 

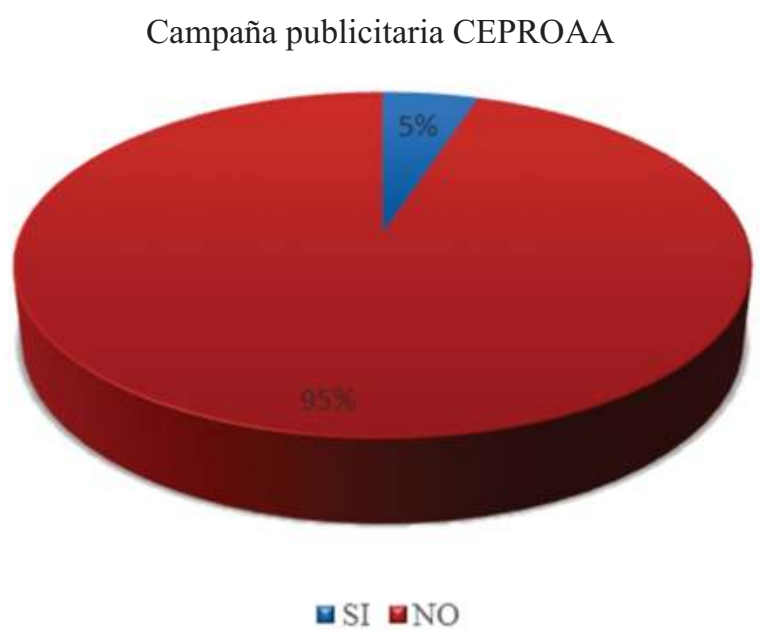

Figura 10. Campaña publicitaria por parte de la Asociación Central de Productores Agropecuarios de AmazonasCEPROAA

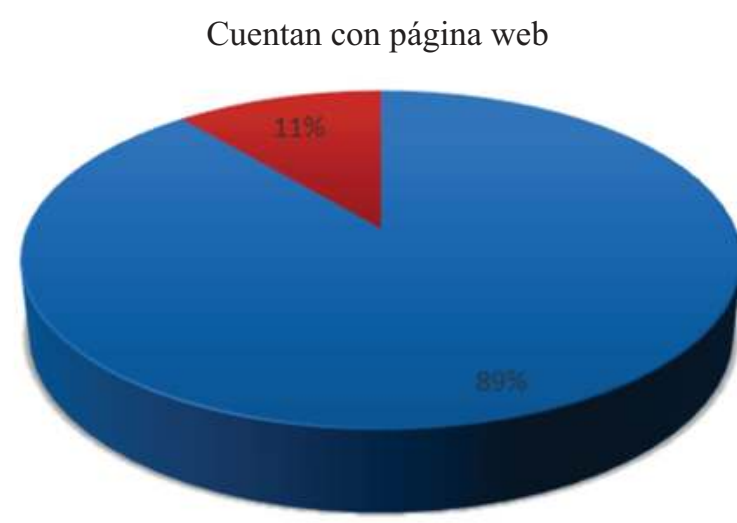

$\mathbf{a S I} \mathbf{a N O}$

Figura 11. Disponibilidad de página web.

\section{DISCUSIÓN}

El 65\% de los socios cuenta con más de 5 años de permanencia en la asociación, el $20 \%$ está entre 3 a 5 años, el $14 \%$ tiene entre 1 y 3 años y el $1 \%$ está entre de 6 meses a 1 año (Figura 1).

Según Palacios (2010), las estrategias cooperativas de las firmas se han convertido en un componente fundamental en el repertorio de la estrategia.

La importancia de los procesos de asociación de productores y búsqueda de actores del comercio justo hacen sostenible su participación en un mercado con potencialidad. Según Ocampo, Ríos y Soria (2012,p. 21)

La asociatividad constituye una opción para todos aquellos agricultores que deseen mejorar su poder de negociación frente a la los proveedores y clientes, buscando el desarrollo de sus asociados

Se puede apreciar, que el $57 \%$ de los socios se dedican más de 8 años a esta actividad, el $28 \%$ de los socios se dedican entre 5 y 8 años, el $14 \%$ de los socios se dedican entre 3 y 5 años y el resto de socios entre 0 y 3 años

(Figura 2).

La asociatividad tiene buena valoración por parte de las familias que producen cacao, el $92 \%$ de la población reconoce que pertenecer a una cooperativa o asociación de productores permite incrementar los ingresos familiares y por tanto, mejorar la calidad de vida (Roberto Mori, 2018)

En el contexto del desarrollo de una cadena de suministro es importante la integración entre todos los actores que intervienen en un proceso productivo de ahí la importancia que exista asociaciones como formas de organización que permitan mejorar la negociación con proveedores y clientes.

Se puede apreciar, que el $52 \%$ de los socios tienen un rendimiento entre 642 a 822 kilos de cacao, el $28 \%$ de los socios tienen un rendimiento entre 823 a 1003 kilos, el $15 \%$ de los socios tienen un rendimiento entre 461 a 641 kilos, el 4\% de los socios tienen un rendimiento entre 280 a 460 kilos y el $1 \%$ de los socios tienen un rendimiento entre 99 a 279 kilos en seco de cacao criollo (Figura 3).

La tendencia internacional de la demanda de cacao presenta para la región Amazonas, un escenario prominente en el mediano plazo. Actualmente el mercado del cacao crece a un ritmo de $3 \%$ en todo el mundo $\mathrm{y}$, según las proyecciones, la demanda mundial está en continuo crecimiento y puede llegar a incrementarse hasta $30 \%$ para 2020 (Beg et al., 2017)

Se sabe que la demanda de cacao a nivel mundial es mayor que la oferta, eso significa una gran oportunidad para todos los productores cacaoteros. En ese sentido es importante el adecuado manejo agronómico, la tecnificación, la mejora de las capacidades de gestión y de la asociatividad de los agricultores.

El sistema de riego más utilizado por los socios es a través de canal de regadío con $68 \%$, seguido de otros sistemas de regadío con $31 \%$ y el $1 \%$ de los socios riegan por aspersión (Figura 4).

Un parámetro principal para evaluar sistemas de riego es la uniformidad de aplicación de agua en la 
superficie del área irrigada, reflejándose directamente en el manejo y desempeño del cultivo, en la calidad y cantidad de los productos, en la eficiencia del uso del agua, en el costo del riego y por tanto de la producción (Bernardo et al., 2009).

Una baja uniformidad de aplicación de agua causa crecimiento desigual de las plantas y en algunos casos contaminación del suelo con sales, lo cual repercute en la degradación del suelo (Cunha et al., 2008).

De lo anterior se debe mencionar que el uso de uno $\mathrm{u}$ otro sistema de riego debe ser evaluado previamente a su uso por lo que se puede mencionar que bajo el escenario actual que tienen los socios de la Central de Productores Agropecuarios de Amazonas (CEPROAA) no necesariamente es el más recomendable por lo que se debe evaluar para cada caso.

La mayor frecuencia de uso de fertilizante es de 1 $\mathrm{kg} / \mathrm{Ha}$ al año con un $61 \%$, el $14 \%$ de los socios fertiliza $2 \mathrm{~kg} / \mathrm{Ha}$, el $19 \%$ de los socios fertiliza entre 1 a $2 \mathrm{~kg}$ por año y el $1 \%$ de los socios utiliza $1 / 2 \mathrm{~kg}$ por año y por último el 5\% no utiliza ningún tipo de fertilizantes (Figura 5).

Las plantas que son eficientes en la absorción y utilización de nutrientes mejoran en gran medida la eficiencia de los fertilizantes aplicados, reduciendo los costos de los insumos, y la prevención de las pérdidas de nutrientes a los ecosistemas (Baligar et al., 2001).

En relación a este tema se debe hacer un estudio previo de suelos para determinar cuáles son las carencias que tienen estos y de acuerdo a estos resultados se debe suministrar los fertilizantes que se necesiten.

Se aprecia que el $75 \%$ de los socios comercialización de otra forma el cacao, el 21\% de los socios lo comercializan en sacos a granel, el $4 \%$ de los socios lo comercializan como cacao procesado (Figura 6).

En el comercio del cacao existen dos mercados. El primero de ellos es el tradicional o de granos convencionales; el segundo corresponde al de granos especializados, en donde se manejan precios diferenciados. El primero es el mercado de los grandes volúmenes, el segundo muestra una alta tasa de crecimiento y su demanda se debe a que los granos -para los consumidores tienen características especiales distintas a los convencionales (Barrientos 2015).

En el mercado nacional la forma de comercialización es en sacos de yute de $69 \mathrm{~kg}$, cacao nibs 227 gr, liquor de cacao 1 litro, barra de chocolate 100gr, manteca de cacao 250 gr, chocolate de taza 90 gr y pasta de cacao 500 gr. Por lo que se debe buscar diversificar la producción para tener mejores ingresos.

El 15\% de los socios participan en ferias, sin embargo el 85\% no participan en ferias (Figura 7).

Las ferias incluyen actividades en las que la oferta y la demanda de diversos productos se concentran en un mismo espacio y además se hacen ruedas de negocio que permiten la posibilidad de generar relaciones comerciales con proveedores y clientes. Además de conocer a la competencia.

El $62 \%$ de los socios opina que es el mercado exterior quien determina el precio del cacao, el 16\% de los socios opinan que es el mayorista, el 14\% de los socios opina que es el acopiador y el $8 \%$ de los socios opina que el productor es quien determina el precio (Figura 8).

En cuanto a los aspectos de fijación de precios internos para el cacao en Colombia, se debe mencionar la implementación de la norma Icontec 1252, ratificada el 28 de mayo de 2003, en ella establece la clasificación y los requisitos que debe cumplir el cacao en grano, destinado a la industrialización para el consumo humano. El proceso de fijación de precios, inicia con el sistema de compra de cacao por calidad y rendimiento y dentro de esto, se fijan los objetivos de fermentación, secado, tamaño, etcétera. Además de estos aspectos, existen otros factores que inciden sobre la fijación de precios, tales como los pactos para la satisfacción de la demanda interna y acuerdos informales informales para la fijación de precios, que se dan en un mercado oligopsónico como el colombiano, entre las dos compañías líderes Compañía Nacional de Chocolates y Casa Luker Dentro de la regulación económica y con el fin de evitar un desabastecimiento de grano para la industria, cuando los precios del mercado son lo suficientemente altos para generar incentivos para exportar el grano, se castigan las exportaciones por medio de cesiones al fondo de estabilización de precios FEPEC en un porcentaje sobre la diferencia entre el precio internacional y el precio de referencia (Roa et al., 2006).

Se puede mejorar los márgenes de ganancia al diferenciar el producto en términos de calidad y darle un valor agregado. De esta manera se extiende la vida útil del producto.

Se puede apreciar, que el $48 \%$ de los socios considera que se debe mejorar los canales de comercialización, el 28\% de los socios considera que se debe mejorar otros, el $15 \%$ de los socios considera que se debe 
mejorar la gestión y el $9 \%$ de los socios piensa que se debe crear una marca (Figura 9).

En el eslabón de comercialización del cacao .Intervienen principalmente las organizaciones de productores y acopiadores de empresas industriales que muchas veces distorsionan los precios. Sus conceptos de calidad y apreciación están sujetos a la variedad de cacao.

El $95 \%$ de los socios mencionan que no se ha realizado ninguna campaña publicitaria por parte de la asociación, por otro lado el $5 \%$ de los socios opinan que si se ha realizado alguna campaña publicitaria (Figura 10).

La importancia de desarrollar campañas publicitarias para lograr posicionar un producto es importante, las consideraciones que se debe tener en cuenta son que medios son más eficientes al momento de hacer publicidad, el costo y la frecuencia de estos.

El 89\% de los socios mencionan que la Asociación Central de Productores Agropecuarios de Amazonas - CEPROAA cuenta con una página web, sin embargo el $11 \%$ de los socios mencionan que no cuentan con una página web (Figura 11).

Es necesario dar a conocer a los socios cuales son los medios con los que cuenta la asociación para que puedan también ellos recomendar la visita a este recurso por parte de los potenciales clientes.

\section{CONCLUSIONES}

En relación al desarrollo de la cadena de suministro para la central de productores agropecuarios de Amazonas (CEPROAA) esta se ha elaborado considerando a todos los involucrados es decir proveedores, centros de producción, nodos de acopio, plantas de procesamiento, depósitos temporales, terminales, canales de comercialización y clientes. De esta forma se tiene identificado a cada uno de los eslabones que participan, de esta manera lo que se busca es compartir información y disminuir los costos totales. Anticipándose a cambios en el mercado y compartiendo riesgos y ganancias entre ellos.

Con respecto a la mejor alternativa de gestión para el flujo de materiales e información esta se tiene que realizar al considerar la disminución de costos totales es decir a utilizar estrategias que permitan la optimización de recursos a través de anticiparse a los cambios de mercado, buscando la flexibilidad en algunos procesos así como considerando los aportes por ejemplo de los proveedores en relación a sugerir la cantidad a considerar al hacer nuevos requerimientos de artículos o a la entrega de materiales en los momentos oportunos que guardan relación con los procesos productivos. Finalmente la mejor manera de mejorar la toma de decisiones es tener un sistema integrado entre todos los miembros que integran la cadena de suministro del cacao. De esta manera se optimiza el manejo de información y se mecaniza los formatos de gestión, así como se tiene control en relación a los tiempos en relación al trámite documentario que se requiere.

En relación a la implementación del enfoque y las estrategias de Supply Chain Management en el manejo del cacao esta tiene que iniciarse sensibilizando a los diferentes miembros de la cadena que la integran, a través de talleres. Lo segundo a tener en cuenta es basarse en modelos exitosos (benchmark), hacer una evaluación, priorizar (identificar oportunidades), identificar el eslabón más débil, establecer la forma de comunicación entre los integrantes, asociarse (buscar sinergias), liderazgo, competencias básicas (cualidades y debilidades) y finalmente tener una política de mejoramiento continuo.

Con respecto al diagnóstico de la central de productores agropecuarios de amazonas (CEPROAA) se debe mencionar que esta cuenta con dos certificaciones (Comercio justoy orgánica). Esta tiene presencia en los distritos de Cajaruro, Bagua Grande y Jamalca en la provincia de Utcubamba y en los distritos de La Peca, Copallin en la Provincia de Bagua. Agrupa en la actualidad a 515 socios entre hombres y mujeres que cultivan café y cacao. Los puntos a mejorar en la parte de operativa son: la ampliación de módulos de fermentación y secado, el arreglo e implementación zarandas eléctricas, el mejoramiento de almacenes. La adquisición de parihuelas para almacenamiento, el cercado perimetral, la compra de carpas y micas, la ampliación de la loza de secado, la compra de equipos e implementación del laboratorio para el control de calidad.

\section{REFERENCIAS BIBLIOGRÁFICAS}

Arboníes, A. (2000). El Cluster del Conocimiento. Nueva Revista De Política Cultura y Arte, (70),57- $\quad 62$. Un ivers i d a d Internacional de la Rioja. España

Ballou, R. (2004). Administración de la cadena de suministro, D.F. México, Quinta edición, Editorial PEARSON.

Bamber, P. y K. Fernández. (2012). Mejora en 
la cadena de valor del cacao orgánico en Perú. Centro para la Globalización, Gobernanza y Competitividadde la Universidad de Duke (CGGC).

Bowersox, D. (2007). Administración y Logística en la cadena de suministros, D . F . México, México. Primera edición, Editorial McGraw- Hill.

Casanovas, A. (2012). Logística Integral, Barcelona, España. Primera edición. Editorial. PROFIT.

CEI. (2010).Asociatividad. Recuperado de http:// www.cei.org.ni/images/file/manual_asocia tiv.pdf.

CODEL. (s.f).Competividad y desarrollo local. Recuperadodehttp://www.uptc.edu.co/expor t/sites/default/facultades/f_economicas/pregra do/administracion/documentos/CODEL.pd f.

Clark, T.; Osterwalder, A. \& Pigneur, Y. (2012). Editado por Megan Lacey. Traducido por Lara Vasquez Caro. Tu Modelo de Negocio. (2da Ed.). España: Editorial Centro Libros.Colburn, F. (2009). América latina: prisionera de los commodities. En: D.F. México,

México. Quinta edición, Editorial PEARSON.

Gottret, M. y M. Lundy. (2007). Gestión de cadenas productivas, Bogotá, C o $10 \mathrm{~m}$ b i a editorial centro internacional de agricultura tropical.

Graziani, L., Ortiz L., Álvarez N., Trujillo A. (2003). Fermentación del cacao en dos diseños de cajas de madera. Agronomía Tropical. 53:175-187.

Herrera Ramírez, Rolando (2012). El cacao en el Perú. Lima, Asociación International Cocoa

Instituto Interamericano de Cooperación para la Agricultura -ICCA- (2006). Protocolo estandarizado de oferta tecnológica para el cultivo del cacao en Perú. Lima, Perú.

Jain, Naresh (2002). Marketing Internacional. México D. F.: International Thomson Editores S.A., 718p.

Jenkins, Rhys (2011). El "Efecto China" en los precios de los productos básicos y en el $\mathrm{v}$ a 1 o $\mathrm{r}$ de las exportaciones de América latina. En: Revista CEPAL, Santiago de Chile, No.103, p. 77-93.

Ministerio de Agricultura (2006). Plan estratégico: Cadena agroproductiva del cacao. Lima:IICA-CICDA-GTZ, 95p.
Mendoza, A., Gallardo, R., Avendaño, C. (2011). El mundo del cacao. Agroproductividad 2:1826. México. Quinta edición, Editorial PEARSON.

Organization -ICCO- (2012). La economía cacaotera mundial: pasado y presente. Londres: International Cocoa Organization, $16 \mathrm{p}$.

Sunil, CH y Meindl, P. (2013). Administración de la cadena de suministro. México. Tercera edición, Editorial PEARSON. 